\title{
Comparison of the biological properties of two strains of Paecilomyces lilacinus (Thom) Samson associated to their antagonistic effect onto Toxocara canis eggs
}

\author{
Gortari, M. C., ${ }^{1,3}$ Galarza, B. C., ${ }^{1,3}$ Cazau, M. C. ${ }^{2}$ and Hours, R. A. ${ }^{1^{*}}$ \\ ${ }^{1}$ Research and Development Centre for Industrial Fermentations (CINDEFI). UNLP; CCT-La Plata, CONICET. School of \\ Science, National University of La Plata. La Plata, Argentina \\ ${ }^{2}$ Institute Spegazzini. School of Natural Science and Museum, National University of La Plata, La Plata, Argentina \\ ${ }^{3}$ Buenos Aires Province Research Commission, Argentina \\ E-mail: hours@biotec.org.ar
}

Received 23 July 2008; received in revised form 25 September 2008; accepted 25 September 2008

\begin{abstract}
Biological control of nematodes by fungi is a promising field of application at large scale. Nematode egg is probably the most resistant stage in the nematode life-cycle, however they are susceptible to colonization and destruction by fungal egg-parasites. Fungal extra-cellular enzymes are able to degrade the main chemical constituents of nematode eggshell and at least they are partly responsible for fungal penetration and/or digestion of the internal tissues. Paecilomyces lilacinus was reported as an ovicidal fungus of Toxocara canis eggs in vitro, a parasite of dogs causing toxocarosis. Two wild-type P. lilacinus LPSC \# 876 and LPSC \# 44 strains, isolated from soil in the main park in the city of La Plata and an agricultural field, respectively, in Argentina, showed antagonism onto $T$. canis eggs. Besides from the isolation source, both strains showed similar hydrolytic profiles activities, ovicidal effect, and grew well on chitin agar, although no chitinolytic activity was detected. Nevertheless, both strains displayed proteolytic and chitinolytic activities in liquid cultures, with maximum values at 14 days of growth. Variable enzyme activities were observed on carboxymethylcellulose and starch agars, whereas the lipolytic activity was poor in both. Percentage of infected $T$. canis eggs was found $65.6 \%$ and $63.2 \%$ for $P$. lilacinus LPSC \# 876 and \# 44 strains respectively. Our results a priori indicate that $P$. lilacinus LPSC \# 876 shows suitable characteristics as a potential agent for biocontrol of $T$. canis.
\end{abstract}

Keywords: Paecilomyces lilacinus, Toxocara canis, nematode eggs, chitinase, protease

\section{INTRODUCTION}

Different kinds of fungi, most of them formerly Deuteromycetes belonging mostly to Ascomycota, show antagonistic effects on eggs of plant - as well as animal and/or human - parasitic nematodes (Lysek and Nigenda, 1989; López-Llorca, 1992; Khan et al., 2003; Huang et al., 2004). The nematode eggshell plays an essential role against adverse environmental conditions and the action of biological and chemical nematicides (López-Llorca et al. 2002; Huang et al., 2004; Khan et al., 2004). Fungal entomopathogenic and mycoparasitic activities were attributed to the penetration of eggshell caused by a combination of mechanical and enzymatic activities over chitin and proteins (Dackman et al., 1989; López-Llorca et al., 2002; Tikhonov et al., 2002). Nematophagous fungi activities on eggshell degradation could be associated to the same mechanisms. In previous works, chitinolytic and proteolytic activities have been detected in fungi with antagonistic activity on nematode eggs. In the last decade, these enzymes have been considered as virulence factors, and some investigations have confirmed their role in the infection mechanism of fungal egg-parasites (Dackman et al., 1989; Bonants et al., 1995; Segers et al., 1996; Tikhonov et al., 2002; Huang et al., 2004; Khan et al., 2004).
Paecilomyces lilacinus (Thom) Samson is a soil filamentous fungus. In vitro studies have demonstrated its antagonistic effects on the eggs from different species of nematodes (Dunn et al., 1982; Araújo et al., 1995; Holland et al., 1999; Basualdo et al., 2000; Olivares-Bernabeu and López-Llorca, 2002; Khan et al., 2003). Most of these studies have been carried out on plant parasitic nematodes, and some strains of $P$. lilacinus have been used as biocontrol agents against these parasites (Cabanillas et al., 1989; Siddiqui and Mahmood, 1996; Holland et al., 1999; Fernández and Juncosa, 2002; Khan et al., 2003). However, there are some nematodes, animal (causative agents of zoonosis) and/or human parasites, which are transmitted by contact with soils contaminated with eggs. Thus, soils containing infective eggs are the main source of infections caused by geohelminths. One of the factors limiting the development and persistence of eggs in the environment is their elimination by antagonistic organisms such as ovicidal fungi (Lysek and Nigenda, 1989; Grønvold et al., 1996). Toxocarosis is a geohelmintic zoonosis resulting from the accidental ingestion of eggs of Toxocara canis, which become infectious after an incubation period of two to five weeks. However, the high resistance of the eggshell allows them to survive for years in soil (Wharton, 1980; Bouchet et al., 2003).

\section{Corresponding author}


Paecilomyces lilacinus interacts in vitro with eggs of $T$. canis by altering their development (Araújo et al., 1995; Basualdo et al., 2000). This fact confirms the observations on the ovicidal ability of $P$. lilacinus on plant parasite nematodes (Morgan-Jones et al., 1987; Holland et al., 1999; Olivares-Bernabeu and López-Llorca, 2002), extending its potential applications as a biocontrol agent to the public health sector. However, to date no studies have been carried on the production of hydrolytic enzymes of $P$. lilacinus related to the infection process of $T$. canis eggs.

Presence of the parasite is neither uniform nor constant in contaminated environments with $T$. canis eggs and it depends on several factors such as: the existence of stray animals, responsible pet ownership, appropriate health education and sanitary control. These factors determine marked epidemiological differences with the main environments (agroecosystems), where the suppression phenomenon caused by fungal egg-parasites has been studied (Gortari et al., 2007). However, ecological conditions constitute one of the main restrictive factors in the progress of the commercial production of fungal biocontrol agents. For this reason, the study of native species belonging to the environments where they are intended to be used is advised (Grønvold et al., 1996; Chen and Chen, 2002).

The aims of the present work were to compare the hydrolytic activities of two wild-type fungal strains $P$. lilacinus LPSC \# 876 and \# 44 isolated form a T. canis contaminated area and from an agricultural soil (no contaminated area) respectively, and their ovicidal activity against $T$. canis eggs.

\section{MATERIAL AND METHODS}

\section{Characteristics of the studied area}

Paseo del Bosque of La Plata is the largest park of the City. This is a traditional park frequented by children where recreational, cultural and sports activities take place. Pets and stray animals (particularly dogs) are frequently found in this area (Gortari et al., 2007).

\section{Soil samples}

Thirty soil samples were collected from the southern sector of the area under study, during the end of spring and beginning of summer with temperatures ranged from $17.8^{\circ} \mathrm{C}$ to $21.1^{\circ} \mathrm{C}$, and relative humidity from $75.6 \%$ to $81.3 \%$. Five hundred grams, up to $10 \mathrm{~cm}$ depth, were randomly taken, separating the upper layer of soil (leaf litter). Samples were kept (never longer than 21 days) in plastic bags at room temperature before processing.

\section{Toxocara canis eggs}

Toxocara canis specimens were obtained by deworming naturally infected puppies. Eggs were extracted from the uterus of adult females of the nematode, treated with $0.1 \%(\mathrm{v} / \mathrm{v}) \mathrm{NaClO}$ (diluted household bleach) and washed repeatedly with sterile distilled water. Eggs were finally resuspended in sterile distilled water at a concentration of $\cong$ $1 \times 10^{3} \mathrm{eggs} / \mathrm{mL}$, and used within $24 \mathrm{~h}$ after extraction. Microscopic observation revealed that most eggs were unembryonated (e.g. 0-1 day old).

\section{Fungus isolation, preservation and identification}

Fungi were isolated using the soil sprinkling technique adapted for the isolation of fungal egg-parasites (Gray, 1984; Chen and Chen, 2002). Soil ( $0.5 \mathrm{~g}$ ) was spread on the surface of Petri dishes containing water-agar $(20 \mathrm{~g} / \mathrm{L})$ supplemented with streptomycin $(100 \mathrm{mg} / \mathrm{L})$ and chlortetracycline $(50 \mathrm{mg} / \mathrm{L})$. Sprinkled plates were inoculated with $1 \mathrm{ml}$ suspension of $T$. canis eggs (ca. $1 \times$ $10^{3} / \mathrm{mL}$ ), placed in plastic bags and incubated at $25^{\circ} \mathrm{C}$ for 3 weeks in the dark. Presence of nematophagous eggparasitic fungi was determined by microscopic observation $(100 \times)$. Isolation of the fungi was carried out by standard microbiological techniques in those areas where eggfungus interaction was detected. The colonies were subcultured on potato-dextrose agar and pure isolates were kept. Taxonomic identification of the $P$. lilacinus strain isolated was based on its macro and microscopic characteristics (Samson, 1974). A representative strain was deposited at the Spegazzini Institute Fungal Type Culture Collection (La Plata, Argentina) and labelled LPSC \# 876.

Paecilomyces lilacinus (LPSC \# 44, Spegazzini Institute Fungal Culture Collection) isolated from an agricultural and egg-uncontaminated soil, province of Buenos Aires, Argentina, was included in the experiments for comparative purposes.

\section{Growth and enzymatic production of $P$. lilacinus in solid media}

Fungal growth and enzymatic production of both strains were determined on agar media supplemented with different substrates.

Control medium: Water agar (15 g/L agar, Difco). Polysaccharolytic activities (Carrillo and Gómez Molina, 1998). 1) Colloidal chitin agar (CCA). Colloidal chitin was prepared from chitin flakes (Sigma Chemical, St. Louis, MO) (Lingappa and Lockwood, 1962). 2) Carboxymethylcellulose agar (CMCA). 3) Mineral saltsstarch agar (SSA).

Proteolytic activities. 1) Casein agar (CA) (Koneman and Roberts, 1985). 2) Gelatine agar (GA) (Kunert et al., 1987). Lipolytic activities. 1) Olive oil agar (OOA) (Kunert and Lýsek, 1987). 2) Tween 80 agar (TA) (Carrillo and Gómez Molina, 1998).

Plates were inoculated and incubated at $25^{\circ} \mathrm{C}$ in the dark. Enzymatic activities were determined according to the corresponding substrate degradation in colonies with an average diameter of $5 \mathrm{~cm}$, regardless of incubation time. All experiments were carried out in triplicates and the error was calculated by standard deviation. The results were interpreted taking into account the ratio 
between the degradation halo radio of substrate and the colony according to the following scale: grade 0 , no degradation; grade 1, degradation only under colony centre; grade 2, degradation is evident only under the whole colony; grade 3 , degradation radio $2-10 \mathrm{~mm}$ larger than that of the colony; grade 4 , degradation radio $10 \mathrm{~mm}$ larger than that of the colony (Kunert et al., 1987).

\section{Ovicidal effect of $P$. lilacinus on $T$. canis eggs}

Drops of $T$. canis egg suspension $\left(\cong 1 \times 10^{3} / \mathrm{mL}\right)$ were laid separately onto the edges of a 10-day old colony of $P$. lilacinus developed on water agar $(20 \mathrm{~g} / \mathrm{L})$. Portions of agar with eggs were taken and placed between slides (with lactophenol solution) for microscopic observation after 10 days culture. Five hundred eggs were examined, and percentage of infected and uninfected eggs (developed and undeveloped) was determined. Ovicidal activity was scored on an arbitrary scale (Lýsek et al., 1982) consisting of four levels: low $(<20 \%$ of infected eggs), intermediate (20 to $50 \%$ of infected eggs), high (50 to $80 \%$ of infected eggs) and very high (> $80 \%$ of infected eggs). As control, T. canis eggs were incubated under the above conditions on water agar with no inoculation. All ovicidal tests were run in triplicates.

\section{Fungal growth in liquid medium and production of raw enzymatic extract}

Paecilomyces lilacinus strains were cultured on potato agar for 7 days at $25^{\circ} \mathrm{C}$. The conidia were suspended in $0.02 \%(\mathrm{v} / \mathrm{v})$ Tween 80 solution. Liquid cultures were carried out in $250 \mathrm{~mL}$-Erlenmeyers flasks containing 100 $\mathrm{ml}$ of minimum medium (MM: $4.56 \mathrm{~g} / \mathrm{L} \mathrm{K}_{2} \mathrm{HPO}_{4}, 2.77 \mathrm{~g} / \mathrm{L}$ $\mathrm{KH}_{2} \mathrm{PO}_{4}$ and $0.5 \mathrm{~g} / \mathrm{L} \mathrm{KCl}$ ) supplemented with $10 \mathrm{~g} / \mathrm{L}$ chitin flakes (Sigma) (Bonants et al., 1995). The flasks were inoculated with $2 \mathrm{~mL}$ suspension of conidia $\left(1 \times 10^{6}\right.$ conidia/mL) and incubated at $27{ }^{\circ} \mathrm{C}$ in the dark, with shaking (120 rpm) for 42 days.

Two flasks per each $P$. lilacinus strain were withdrawn every week. Fungal biomass was separated by centrifugation at $4{ }^{\circ} \mathrm{C}$ and culture supernatant was collected by filtration $(0.45 \mu \mathrm{m}$ pore size EO4WP04700, MSI, USA), and kept on an ice-water bath following by freezing at $-20{ }^{\circ} \mathrm{C}$ until analyzed.

\section{Enzymatic activities of crude fungal culture filtrates on agar media}

A semi-quantitative screening of protease and chitinase activities was carried out in both strains of $P$. lilacinus for different culture periods. Proteolytic activity was assessed on $\mathrm{CA}$ and GA and chitinolytic activity was assessed on CCA with the following concentrations $(\mathrm{g} / \mathrm{L})$ of colloidal chitin: 2.5, 5.0, 7.5 and 10. Twenty milliliters of each medium were placed on each Petri dish. After solidification of the medium 8-mm diameter holes (3/plate) were made. One hundred microliters of crude fungal filtrate, corresponding to each culture period of $P$. lilacinus, were placed in each hole (in triplicates). Plates were incubated at $25^{\circ} \mathrm{C}$ and the size of the degradation halo caused by enzymatic activity was recorded until no size changes were observed (Zou et al., 2002).

\section{RESULTS}

\section{Isolation and identification of a native $P$. lilacinus}

A total of 105 fungal wild-type colonies were isolated from soil baited with $T$. canis eggs technique above described. The following genera were identified: Acremonium, Aspergillus, Chrysosporium, Fusarium, Humicola, Mortierella, Paecilomyces and Penicillium, as previously reported (Gortari et al., 2007).

Colonies attributable to $P$. lilacinus on the basis of their appearance and coloration on potato dextrose agar were selected. Their taxonomic identification was confirmed on the basis of its macroscopic and microscopic characteristics on malt agar, according to Samson (Samson, 1974). Among the main characteristics: a) colony diameter between $5-7 \mathrm{~cm}$ after 15 days, at $25^{\circ} \mathrm{C}$; b) velvety colonies with a whitish coloration turning pinkish and then purple upon sporulation; c) microscopic observation revealed verticillate branches with long and spindle-shaped phialides originating small, spherical to ellipsoidal conidia in divergent chains.

\section{Growth and enzymatic production of $\boldsymbol{P}$. lilacinus on agar media}

Paecilomyces lilacinus strains LPSC \# 876 and LPSC \# 44 were able to grow in all agar media tested, as well as in water agar. Table 1 details the enzymatic activities assayed for both strains displaying similar qualitative and semi quantitative behaviour against different substrates tested. Positive enzymatic activities were detected against: polysaccharides (CMCA and SSA), proteins (GA and CA) and lipids (OOA and TA). Conversely, chitinolytic activity in agar was not detected in either case.

Table 1: Enzyme activities of $P$. lilacinus (strains LPSC \# 876 and LPSC \# 44) on agar media

\begin{tabular}{|c|c|c|c|}
\hline \multirow[b]{2}{*}{ Activity tested } & \multirow[b]{2}{*}{$\begin{array}{l}\text { Solid } \\
\text { media }\end{array}$} & \multicolumn{2}{|c|}{ P. Iilacinus strain } \\
\hline & & LPSC \# 876 & $\begin{array}{l}\text { LPSC \# } \\
44\end{array}$ \\
\hline \multirow[t]{3}{*}{ Polysaccharolytic } & CCA & 0 & 0 \\
\hline & CMCA & 4 & 3 \\
\hline & SSA & 2 & 2 \\
\hline \multirow[t]{2}{*}{ Proteolytic } & GA & 3 & 4 \\
\hline & CA & 3 & 4 \\
\hline \multirow[t]{2}{*}{ Lipolytic } & OOA & 2 & 2 \\
\hline & TA & 2 & 3 \\
\hline
\end{tabular}

CCA: colloidal chitin agar. CMCA: carboxymetilcellulose agar. SSA: mineral salts-starch agar. GA: gelatine agar. CA: casein agar. OOA: olive oil agar. TA: Tween 80 agar. Numerals give the degree of the corresponding enzyme activity in an arbitrary scale. See Material and Methods for calculation procedure. 


\section{Ovicidal effect of $P$. lilacinus on $\boldsymbol{T}$. canis eggs}

Parasitism of $T$. canis eggs by $P$. lilacinus strains LPSC \# 44 as well as LPSC \# 876 is shown in Table 2. Percentage of infected, uninfected developed and uninfected undeveloped eggs of $P$. lilacinus LPSC \# 876 was $65.6,22.2$ and $12.2 \%$, respectively. In the case of LPSC \# 44, the percentages were 63.2, 22.0 and 14.8\% for the same experiment, respectively. In this paper, eggs which were invaded by hyphae and with the embryo completely destroyed are considered infected (Figure 1). Uninfected embryonated eggs showed normal development up to viable larval stages (Figure $2 \mathrm{~A}$ ). Most of undeveloped eggs did not show any morphological changes during the incubation period and remained uninfected (Figure $2 \mathrm{~B}$ ). On the other side, most of the uninfected eggs (87\%) used as control displayed development to larval stages. The two strains tested showed similar ovicidal efficiency against $T$. canis eggs.

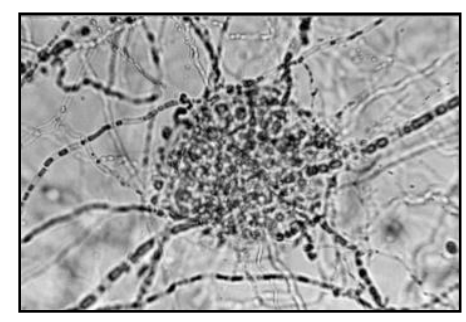

Figure 1: $T$. canis infected egg invaded by $P$. lilacinus hyphae showing its content completely destroyed. Bar $=25 \mu \mathrm{m}$

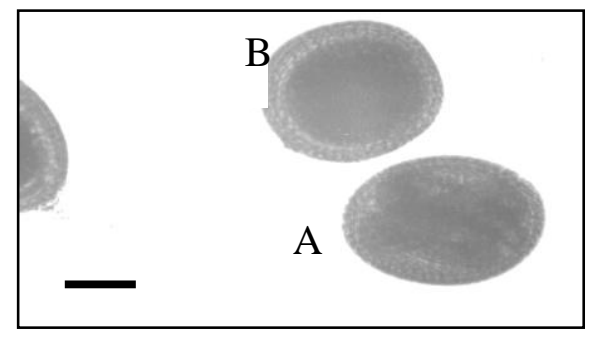

Figure 2: T. canis uninfected eggs. A: developed. B: undeveloped. Bar $=25 \mu \mathrm{m}$

\section{Growth in liquid medium and production of crude enzymatic extract}

Paecilomyces lilacinus strains LPSC \# 876 and LPSC \# 44 developed in liquid media in presence of chitin flakes and produced proteolytic and chitinolytic enzyme activities. Determination of crude extract proteolytic activities was estimated by the diameter of degradation halo size in agar media. The highest proteolytic activity in the filtrates was detected ten days after incubation corresponding to 2 weeks of culture, with a $3 \mathrm{~cm}$ diameter degradation halo both on CA and on GA. No further changes in the halo size were recorded after 10 days of incubation.
Chitinolytic activity was detected only in $10 \mathrm{~g} / \mathrm{L} \mathrm{CCA}$ plates. The highest activity of the filtrates was detected after 7 days incubation (corresponding to 2 weeks of culture), with a $2 \mathrm{~cm}$ diameter degradation halo. No changes were observed after longer incubation periods.

Table 2: Ovicidal activity of $P$. lilacinus (strains LPSC \# 876 and LPSC \# 44) on T. canis eggs. Average $\pm S D, n=500$. See Material and Methods for experimental details

\begin{tabular}{|c|c|c|c|}
\hline \multirow[b]{2}{*}{ Inoculation } & \multirow[b]{2}{*}{$\begin{array}{l}\text { Stage of } T . \\
\text { canis eggs }\end{array}$} & \multicolumn{2}{|c|}{ P. lilacinus strain } \\
\hline & & $\begin{array}{l}\text { LPSC \# } \\
876\end{array}$ & $\begin{array}{l}\text { LPSC \# } \\
44\end{array}$ \\
\hline \multirow{3}{*}{ Yes } & Infected & $\begin{array}{l}328 \pm \\
20\end{array}$ & $316 \pm 17$ \\
\hline & $\begin{array}{l}\text { Uninfected } \\
\text { developed }\end{array}$ & $111 \pm 7$ & $110 \pm 6$ \\
\hline & $\begin{array}{l}\text { Uninfected } \\
\text { undeveloped }\end{array}$ & $61 \pm 4$ & $74 \pm 4$ \\
\hline No (control) & $\begin{array}{l}\text { Developed } \\
\text { Undeveloped }\end{array}$ & $\begin{array}{l}435 \pm 16 \\
65 \pm 2\end{array}$ & \\
\hline
\end{tabular}

\section{DISCUSSION}

Nematode suppression has been studied mainly in agricultural soils. It is caused by fungal egg, female and/or cyst fungal parasites. Presence of these fungi is independent of the nematode population. However, a certain level of predation, as an adaptive response of the fungal population to ecological niches with eggs and cysts of nematodes, has been demonstrated (Chen and Chen, 2002). This association has not been proven for fungi with antagonistic effect on $T$. canis eggs. This may be explained by the ecological and epidemiological characteristics of contaminated soils by this parasite (Lysek and Nigenda, 1989). Nevertheless, the use of $T$. canis eggs as a bait allowed the isolation of egg-parasitic nematophagous fungi from the soil of Paseo del Bosque of La Plata City (a public recreational area contaminated with Toxocara spp eggs). Among these fungi the species $P$. lilacinus, known due to its antagonist activity on nematode eggs, was isolated and identified.

Adaptability to the environment and production of certain extracellular enzymes that participate in the infection process are among the main characteristics for selecting a potentially appropriate fungus to be used as biocontrol agent (Barranco-Florido et al., 2002; OlivaresBernabeu and López-Llorca, 2002). The eggshell of $T$. canis eggs consists of three layers: an outer protein layer, a middle layer formed by a chitin-protein complex and an inner lipid layer (Wharton, 1980). Therefore, a preselection of fungal-egg parasites with activity on $T$. canis eggs may be based on the production of proteases and chitinases. Kunert et al. (1982) studied the polysaccharolytic activity on a series of ovicidal fungi, and cellulolytic activity was detected on $80 \%$ of the fungi while $84 \%$ of the fungi displayed amylolytic activity. However, no correlation was found between these activities and the 
ovicidal capability. Further studies on chitinolytic fungal activity revealed that $73-92 \%$ of the strains degraded different kinds of chitin. However, the percentage depends on the kind of chitin used as substrate (Kunert et al., 1985), but the enzymatic activity correlated positively with the ovicidal activity. Dackman et al. (1989) could not establish a direct relationship between the chitinolytic activity and the ovicidal activity, but they concluded that the latter activity is required to develop the infection process. Olivares-Bernabeu \& López-Llorca (2002) found similar results studying the parasitism of Meloidogyne javanica eggs by $P$. lilacinus.

The fungal-egg parasite wild-type strains of $P$ lilacinus characterized in this study did not show chitinase activity on agar media. However, it is clear that both strains are capable of using chitin as substrate since they have a bigger growth on chitin agar than on water agar. Also, both strains showed cellulolytic and amylolytic activities. High proteolytic activities detected for both strains on gelatine and casein can be related to the basic role of proteases, which together with chitinases were responsible for the alteration of $T$. canis eggs. However, the lipolytic activity detected for both strains was low, agreeing with the results obtained by Olivares-Bernabeu \& López-Llorca (2002).

Proteolytic and chitinolytic activities in crude culture filtrates were evident in the first week of culture, reaching their highest values at 14 days culture. Then, proteolytic activity showed lower values which remained constant during the whole period of culture. Chitinolytic activity was not detected in all samples taken after 14 days of culture. Bonants et al. (1995) found the highest proteolytic activity at approximately 10 days of cultivation and demonstrated the participation of a purified protease of $P$. lilacinus in the destruction of Meloidogyne hapla eggs. Further studies related directly the production of both enzymes to the destruction of nematode eggs. Tikhonov et al. (2002) purified and characterized chitinases from fungal-egg parasites (Verticillium chlamydosporium and $V$. suchlasporium) for the first time. They attributed the destruction of Globodera pallida eggs by the joint action of proteases and chitinases. These hypothesis was confirmed by Khan et al. (2004) assessing the enzymatic action of purified proteases and chitinases of $P$. lilacinus on $M$. javanica eggs. These authors observed deeper structural changes in the eggshell under the simultaneous action of both enzymes than when they were used separately. The lack of agreement among other studies regarding the chitinolytic activity might be attributed to the differences and varieties of fungal species tested, differences in the culture conditions and the substrate used as inducer because of different procedures for colloidal chitin preparation.

The ovicidal effect on unembryonated $T$. canis eggs was similar in both strains. Basualdo et al. (2000) studied the interaction of the LPSC \# 44 strain with $T$. canis eggs (at the same developmental stage used in the present study but under different experimental conditions) and detected $80.9 \%$ of infected eggs. Araújo et al. (1995) found that $P$. lilacinus infected $76 \%$ of embryoned $T$. canis eggs. The strains of $P$. lilacinus used in the present work and in the aforementioned studies are considered as having high ovicidal capability (Lýsek et al., 1982). The differences found in the percentage of infected eggs may be attributed to the different experimental conditions used to study the interaction, to individual differences between the $P$. lilacinus strains assayed and to the developmental stages of the eggs.

The knowledge of the factors affecting the natural control of the nematodes is crucial for the application of the biocontrol agents in situ, and further studies in the field are required in order to confirm the in vitro experimental results. Understanding these factors is fundamental, since the selection of a possible biocontrol agent should be based on its adaptability to the environment in which it is intended to be used, rather than on the simplicity of isolation and facility of maintenance under laboratory conditions (Gray, 1983).

Paecilomyces lilacinus is commonly present in soils. Nevertheless, the previous adaptation to an environment contaminated with eggs potentially pathogenic for humans, and the characteristics expressed in vitro, highlight the isolation of a native $P$. lilacinus as a possible local biocontrol agent for $T$. canis eggs. In addition to infecting nematode eggs, $P$. lilacinus has been reported to infect humans and animals (Khan et al., 2003). Therefore, potential toxicity of $P$. lilacinus LPSC \# 876 (i.e. testing for paecilotoxin production) would be carried out even though it is not compulsory for local product registration. Further studies on the distinctive fungal hydrolytic activities are in progress in our laboratory in order to contribute to the determination of the key events on the nematode egg parasitism.

\section{REFERENCES}

Araújo, J.V., Santos, M.A. and Ferraz, S. (1995). Efeito ovicida de fungos nematófagos sobre ovos embrionados de Toxocara canis. Arquivo Brasileiro de Medicina Veterinaria e Zootecnia 47(1): 37 - 42 (in Portuguese).

Barranco-Florido, J.E., Alatorre-Rosas, R., GutiérrezRojas, M., Viniegra-González, G. and SaucedoCastañeda, G. (2002). Criteria for the selection of strains of entomopathogenic fungi Verticillium lecanii for solid state cultivation. Enzyme and Microbial Technology 30: 910 - 915.

Basualdo, J.A., Ciarmela, M.L., Sarmiento, P.L. and Minvielle, M.C. (2000). Biological activity of Paecilomyces genus against Toxocara canis eggs. Parasitology Research 86: 854 - 859.

Bonants, P.J.M., Fitters, P.F.L., Thijs, H., den Belder, E., Waalwijk, C. and Henfling, J.W.D.M. (1995). A basic serine protease from Paecilomyces lilacinus with biological activity against Meloidogyne hapla eggs. Microbiology 141: 775 - 784

Bouchet, F., Araújo, A., Harter, S., Miranda Chaves, S., Duarte, A.N., Monnier, J.L. and Ferreira, L.F. (2003). Toxocara canis (Werner, 1782) eggs in the pleistocene site of Menez-Dregan, France $(300,000$ 
500,000 years before present). Memórias do Instituto Oswaldo Cruz 98 (Suppl. I): 137 - 139.

Cabanillas, E., Barker, K.R. and Nelson, L.A. (1989). Growth of isolates of Paecilomyces lilacinus and their efficacy in biocontrol of Meloidogyne incognita on tomato. Journal of Nematology 21(2): 164 - 172.

Carrillo, L. and Gómez Molina, S.E. (1998). Producción de quitinasa por una cepa de Streptomyces griseoruber aislada de la rizósfera de caña de azúcar. Revista Argentina de Microbiología 30: 73 - 78 (in Spanish)

Chen, F. and Chen, S. (2002). Mycoflora in cyst, females, and eggs of the soybean cyst nematode in Minnesota. Applied Soil Ecology 19: 35 - 50.

Dackman, C., Chet, I. and Nordbring-Hertz, B. (1989). Fungal parasitism of the cyst nematode Heterodera schachtii infection and enzymatic activity. FEMS Microbiology and Ecology 62: 201 - 208.

Dunn, M.T., Sayre, R.M., Carrell, A. and Wergin, W.P. (1982). Colonization of nematode eggs by Paecilomyces lilacinus (Thom) Samson as observed with scanning electron microscope. Scanning Electron Microscopy 3: 1351 - 1357.

Fernández, C. and Juncosa, R. (2002). Biopesticidas: ¿La agricultura del futuro? Phytoma 141: 14-19 (in Spanish)

Gortari, C., Cazau, C. and Hours, R. (2007). Hongos nematófagos de huevos de Toxocara canis en un paseo público de La Plata, Argentina. Revista Iberoamericana de Micología 24: 24 - 28 (in Spanish).

Grønvold, J., Henriksen, SAa., Larsen, M., Nansen, P. and Wolstrup, J. (1996). Biological control aspects of biological control - with special reference to arthropods, protozoans and helminths of domesticated animals. Veterinary Parasitology 64: 47 -64 .

Gray, N.F. (1983). Ecology of nematophagous fungi: distribution and habitat. Annals of Applied Biology 102: $501-509$.

Gray, N.F. (1984). Ecology of nematophagous fungi: Methods of collection, isolation and maintenance of predatory and endoparasitic fungi. Mycopathologia 86: $143-153$.

Holland, R.J., Williams, K.L. and Khan, A. (1999). Infection of Meloidogyne javanica by Paecilomyces lilacinus. Nematology 2: 131 - 139.

Huang, X., Zhao, N. and Zhang, K. (2004). Extracellular enzymes serving as factors in nematophagous fungi involved in infection of the host. Research in Microbiology 155: 811 - 816.

Khan, A., Williams, K. and Nevalainen, H. (2003) Testing the nematophagous biological control strain Paecilomyces lilacinus 251 for paecilotoxin production. FEMS Microbiology Letters 227: 107 111.

Khan, A., Williams, K.L. and Nevalainen, H.K.M. (2004). Effects of Paecilomyces lilacinus protease and chitinase on the eggshell structures and hatching of
Meloidogyne javanica juveniles. Biological Control 31: $346-352$.

Koneman, E.W. and Roberts, G.D. (1985). Practical Laboratory Mycology, $3^{\text {rd }}$ edn. Williams \& Wilkins. Baltimore. p. $207-218$.

Kunert, J. and Lýsek, H. (1987). Lipolytic activity of ovicidal soil fungi. Biologia (Bratislava) 42: 285 - 293.

Kunert, J., Zemek, J., Augustín, J. and Kuniak, E. (1987). Proteolytic activity of ovicidal soil fungi. Biologia (Bratislava) 42: 695 - 705.

Kunert, J., Zemek, J., Augustín, J., Kuniak, E. and Chalupová, V. (1985). Chitinolytic activity of ovicidal soil fungi. Biologia (Bratislava) 40: 1157 - 1165.

Kunert, J., Zemek, J., Augustín, J., Kuniak, E. and Lýsek, H. (1982). Polysaccharide-hidrolyzing activity of ovicidal fungi. Biologia (Bratislava) 37: 291 - 299.

Lingappa, Y. and Lockwood, L. (1962). Chitin media for selective isolation and culture of actinomycetes. Phytopathology 52: 317 - 323.

López-Llorca, L.V. (1992). Los hongos parásitos de invertebrados y su potencial como agentes de control biológico. Revista lberoamericana de Micología 9: 17 -22 (in Spanish).

López-Llorca, L.V., Olivares-Bernabeu, C., Salinas, J., Jansson, H-B. and Kolattukudy, P.E. (2002). Prepenetration events in fungal parasitism of nematode eggs. Mycological Research 106: 499 - 506.

Lýsek, H., Fassatiová, O., Pineda Cuervo, N. and Hernández Lorenzo, N. (1982). Ovicidal fungi in soil of Cuba. Folia Parasitologica (Praha) 29: 265 - 270.

Lysek, H. and Nigenda, G. (1989). Capacidad de autodeshelmintizacion del suelo. Salud Pública de México 31: 763 - 771 (in Spanish).

Morgan-Jones, G. and Rodriguez-Kabana, R. (1987). Fungal biocontrol for the management of nematodes. In: Vistas on Nematology. Veech, J.A. and Dickson, D.W. (eds.). Society of Nematologists, Maryland, p. $94-99$.

Olivares-Bernabeu, C. and López-Llorca, L.V. (2002). Fungal egg-parasites of plant-parasitic nematodes from Spanish soils. Revista Iberoamericana de Micología 19: 104 - 110.

Samson, R.A. (1974). Paecilomyces and some allied hyphomycetes. Stud. Mycol. 6: 1 - 119.

Segers, R., Butt, T.M., Kerry, B.R., Beckett, A. and Peberdy, J.F. (1996). The role of the proteinase VCP1 produced by the nematophagous Verticillium chlamydosporium in the infection process of nematode eggs. Mycological Research 100: 421 428.

Siddiqui, Z.A. and Mahmood, I. (1996). Biological control of plant parasitic nematodes by fungi: a review. Bioresource Technology 58: 229 - 239.

Tikhonov, V.E., López-Llorca, L.V., Salinas, J. and Jansson, H-B. (2002). Purification and characterization of chitinases from the nematophagous fungi Verticillium chlamydosporium and V. suchlasporium. Fungal Genetics and Biology 35: $67-78$. 
Mal. J. Microbiol. Vol 4(2) 2008, pp. 35- 41

Wharton, D. (1980). Nematode egg-shells. Parasitology 81: $447-463$

Zou, X., Nonogaki, H. and Welbaum, G.E. (2002). A gel diffusion assay for visualization and quantification of chitinase activity. Molecular Biotechnology 22: 19 23. 